\title{
A standard stellar library for evolutionary synthesis
}

\section{Metallicity calibration}

\author{
P. Westera ${ }^{1}$, T. Lejeune ${ }^{2}$, R. Buser ${ }^{1}$, F. Cuisinier ${ }^{3}$, and G. Bruzual ${ }^{4}$ \\ 1 Astronomisches Institut der Universität Basel, Venusstrasse 7, 4102 Binningen, Switzerland \\ e-mail: westera@astro.unibas.ch, buser@astro.unibas.ch \\ 2 Observatório Astronómico da Universidade de Coimbra, Portugal \\ e-mail: lejeune@mat.uc.pt \\ 3 Depto. de Astronomia, Universidade Federal do Rio de Janeiro, Brazil \\ e-mail: cuisinie@sun1.ov.ufrj.br \\ 4 Centro de Investigaciones de Astronomía, Mérida, Venezuela \\ e-mail: bruzual@cida.ve
}

Received 17 July 2001 / Accepted 19 October 2001

\begin{abstract}
We extend the colour calibration of the widely used BaSeL standard stellar library (Lejeune et al. 1997, 1998) to non-solar metallicities, down to $[\mathrm{Fe} / \mathrm{H}] \sim-2.0$ dex. Surprisingly, we find that at the present epoch it is virtually impossible to establish a unique calibration of UBVRIJHKL colours in terms of stellar metallicity $[\mathrm{Fe} / \mathrm{H}]$ which is consistent simultaneously with both colour-temperature relations and colour-absolute magnitude diagrams (CMDs) based on observed globular cluster photometry data and on published, currently popular standard stellar evolutionary tracks and isochrones. The problem appears to be related to the longstanding incompleteness in our understanding of convection in late-type stellar evolution, but is also due to a serious lack of relevant observational calibration data that would help resolve, or at least further significant progress towards resolving this issue. In view of the most important applications of the BaSeL library, we here propose two different metallicity calibration versions: (1) the "WLBC 99" library, which consistently matches empirical colour-temperature relations and which, therefore, should make an ideal tool for the study of individual stars; and (2), the "PADOVA 2000" library, which provides isochrones from the Padova 2000 grid (Girardi et al. 2000) that successfully reproduce Galactic globular-cluster colour-absolute magnitude diagrams and which thus should prove particularly useful for studies of collective phenomena in stellar populations in clusters and galaxies.
\end{abstract}

Key words. catalogs - stars: abundances - stars: atmospheres - stars: fundamental parameters

\section{Introduction}

As present grids of theoretical spectral energy distributions (SEDs) suffer from intrinsic inhomogeneities and incompleteness and show large systematic discrepancies with empirical calibrations due to unavailable molecular opacity (see Lejeune et al. 1997, hereafter Paper I), we have undertaken the construction of a comprehensive combined library of realistic stellar flux distributions. Empirical $T_{\text {eff-colour relations in } U B V R I J H K L}$ photometry are used to adjust the spectra using an algorithm developed by Cuisinier et al. (see Buser \& Kurucz 1992, Paper I).

Send offprint requests to: R. Buser, e-mail: buser@astro.unibas.ch
The current state of the art is the following: The semi-empirical BaSeL (Basel Stellar Library) 2.2 SED library (Lejeune et al. 1998, hereafter Paper II) has been widely used successfully in different areas (Bruzual et al. 1997; Kauffmann \& Charlot 1998; Lastennet et al. 1999; González Delgado et al. 1999; Origlia et al. 1999; Leitherer et al. 1999; Bruzual 1999; Brocato et al. 2000; Maraston \& Thomas 2000; Barmby \& Huchra 2000; Liu et al. 2000; Lastennet et al. 2000; Lotz et al. 2000; Mollá \& GarcíaVargas 2000; Nikolaev \& Weinberg 2000; Kong et al. 2000; Lee et al. 2000; Marleau et al. 2000; Johnson et al. 2000; Lastennet et al. 2001; Kotilainen et al. 2001; Fricke et al. 2001). However, as a result of being calibrated from solar metallicity data only, BaSeL 2.2 still has its weaknesses at low metallicities $([\mathrm{Fe} / \mathrm{H}]<-1)$, especially in the 
ultraviolet $(U-B)$ and the infrared $(V-K, J-H, H-K$, $J-K, K-L)$. In these colours and at these lower metallicities, synthetical globular cluster CMDs appear too blue using the BaSeL 2.2 semi-empirical library. Furthermore, the transition between dwarfs and giants produces a discontinuity in some colours (for more details, see Papers I and II as well as T. Lejeune's thesis 1997).

The purpose of the present paper is to remove the weaknesses of the BaSeL 2.2 semi-empirical library by extending the colour-calibration to low metallicities, and to create a library that reproduces empirical colourtemperature relations and globular cluster CMDs (using existing grids of isochrones) at all metallicities.

The outline of the paper is the following: in Sect. 2, we discuss the compilation and properties of the calibration data, i.e. globular cluster CMDs and empirical colour- $T_{\text {eff }}$ relations. In Sect. 3, we briefly describe the calibration algorithm and the changes made relative to the previous algorithm (described in Paper I). As a result, we present the BaSeL 3.1 "WLBC 99" SED library, which is able to reproduce empirical colour-temperature relations in all ( $U B V R I J H K L)$ colours, and is therefore projected to be a powerful tool for studies of individual stars. Unfortunately, the library doesn't provide any improvements in the representation of globular cluster CMDs, and it even proved impossible to provide a library that satisfies both requirements at the same time. As a pragmatic solution, we also produce an application-oriented library, the BaSeL "Padova 2000" library, which, if used along with the Padova 2000 isochrones, successfully reproduces globular cluster CMDs at all levels of metallicity; this version of the library is presented and discussed in Sect. 4 . The conclusions are summarised in Sect. 5, where an outlook on future work and on a first application can also be found.

A more detailed description of this work is given in Westera (2001).

\section{Compilation and discussion of calibration data}

In order to carry out the colour-calibration in a metallicity-dependent way, we collected empirical photometry from the literature of the best-studied Galactic globular clusters, spanning wide ranges in metallicity and evolutionary stages. The clusters used were 47 Tuc, M 5, M 3 , NGC 6397 and M 92, with the respective $[\mathrm{Fe} / \mathrm{H}]$-values of $-0.70,-1.11,-1.34,-1.82$, and -2.16 (Carretta \& Gratton 1997). To reach the best possible coverage of the multi-colour $(U B V R I J H K L)$ - magnitude - diagram of each cluster, the photometry was combined from numerous sources summarised in Table 1. Where necessary, the photometry was brought onto the Johnson-Cousins system using colour-colour relations by Bessell and collaborators (Bessell 1979, 1983; Bessell \& Weiss 1987; Bessell $\&$ Brett 1988). Where no fiducial lines were given, they were drawn by eye. In some colours (mainly $B-V$ and $V-I$ ), different sources were found for the same part of the CMD of the same cluster. The fiducials derived from or given by such sources usually didn't differ from each other by more than a few hundredths of a magnitude.

In order to put these colour-magnitude diagrams onto the intrinsic system, the $E_{B-V}$ and $(m-M)_{V}$ values from the Harris online catalog of globular cluster parameters (Harris 1996) were used. The adopted distance scale is based on the luminosity level of the horizontal branch (HB), where the absolute magnitude of the HB was derived from an empirical, metallicity-dependent relation $\left(M_{V}(\mathrm{HB})=0.15[\mathrm{Fe} / \mathrm{H}]+0.80\right)$. Harris estimates that the absolute uncertainty of the predicted $M_{V}(\mathrm{HB})$ is of the order of $0.1-0.2^{\mathrm{m}}$, in the non-extreme cases of the used clusters more likely $0.1^{\mathrm{m}}$. For the extinction coefficients, he gives an uncertainty of $\Delta E_{B-V}=0.1 E_{B-V}$. This shouldn't pose any problems, because we used clusters with extinction coefficients of only a few hundredths of a magnitude (only NGC 6397 has an $E_{B-V}$ of 0.18 ). The result are the fiducials shown in Figs. 1 and 2 (only the main sequence, the subgiant branch and the red giant branch are shown because the other parts of the CMD weren't used for the calibration).

Two properties of the empirical CMDs are very striking. First, there are large gaps in the data. Measurements down to a few magnitudes below the main sequence turnoff exist only in $B V I$. In the far infrared, hardly any data at all are available. In order to create a sufficient photometric basis for population synthesis, more work clearly needs to be done on the observational side, in order to improve results based on either observational or synthetic spectra.

The second striking point about the CMDs shown in Figs. 1 and 2 is the fact that their behaviour with increasing metallicity isn't as systematic or monotonous as one would expect. The colours of the RGB at a given $(V)$ magnitude can differ up to $\sim 0.1^{\mathrm{m}}$ from any trend with $[\mathrm{Fe} / \mathrm{H}]$. The scatter around trends with metallicity is not of the same amplitude for all colours. While in $U-B$, $B-V$, and $R-I$ it is the most pronounced, $V-I$ behaves much more systematically with $[\mathrm{Fe} / \mathrm{H}]$, as is also seen in homogeneous databases like the one from Saviane et al. $(2000)^{1}$. Part of the scatter (a few hundredths of a mag, see above) can be explained by the heterogeneity of the database which however cannot account for the entire scatter in those colours where it is observed. Obviously, there are parameters apart from age and metallicity that govern the appearance of the CMD. It is therefore more than amazing how in the literature, one often finds perfect agreement between synthetic isochrones and empirical CMDs for whole sets of globular clusters at the same time.

From these CMDs, combined multi-colour $(U B V R I J H K L)-[\mathrm{Fe} / \mathrm{H}]-T_{\mathrm{eff}}-\log g$ relations were synthesised, using the $T_{\text {eff }}-(V-K)$ relation from the BaSeL 2.2 library. This relation incorporates Ridgway et al. (1980) complemented with the differential properties

\footnotetext{
1 In the infrared colours, there were barely enough data to derive a trend, but certainly not enough to determine the amplitude of a possible scatter around it.
} 

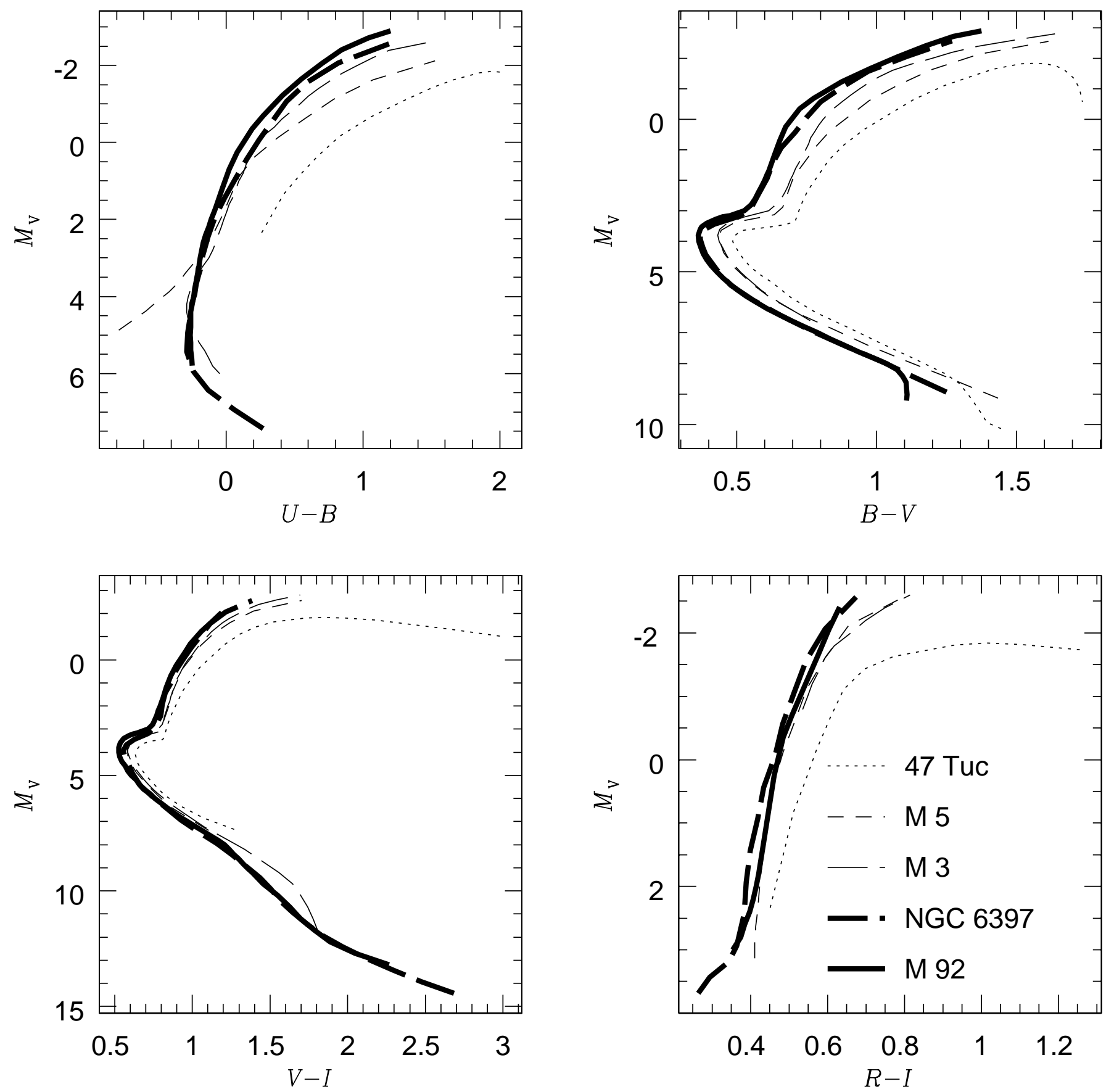

Fig. 1. Empirical $(U B V R I)$ fiducial lines in the CMD of the globular clusters 47 Tuc, M 5, M 3, NGC 6397 and M 92.

in $T_{\text {eff }}$ for solar metallicity of the original (uncalibrated) grid, as the latter is the only relation which covers the entire range in temperature. This resulting relation was used for all metallicities, because the $V-K$ colour is expected to be metallicity insensitive (von Braun et al. 1998; Alonso et al. 1999). Finally, $\log g$ values were added to the calibration files, using empirical $T_{\text {eff }}-\log g$ relations for red giants from Cohen, Frogel and Persson (Cohen et al. 1978; Frogel et al. 1981, 1983, [Fe/H]-dependent) and for dwarfs from Angelov (1996, [Fe/H]-independent).
We could only produce colour-temperature relations in the ranges specified in Table 2. For giants, the ranges include the entire RGB (except in $K-L$ ). Outside these ranges, we used the differential properties with regard to $T_{\text {eff }}$ of the semi-empirical (BaSeL 2.2) grid, as there was no other information available (neither empirical nor theoretical) about the behaviour of these colours for these temperatures. In $K-L$ for all stars, and for dwarfs in $R-I, V-K, J-H, H-K$, and $J-K$, where no observed data were available, but which are needed to complete the 

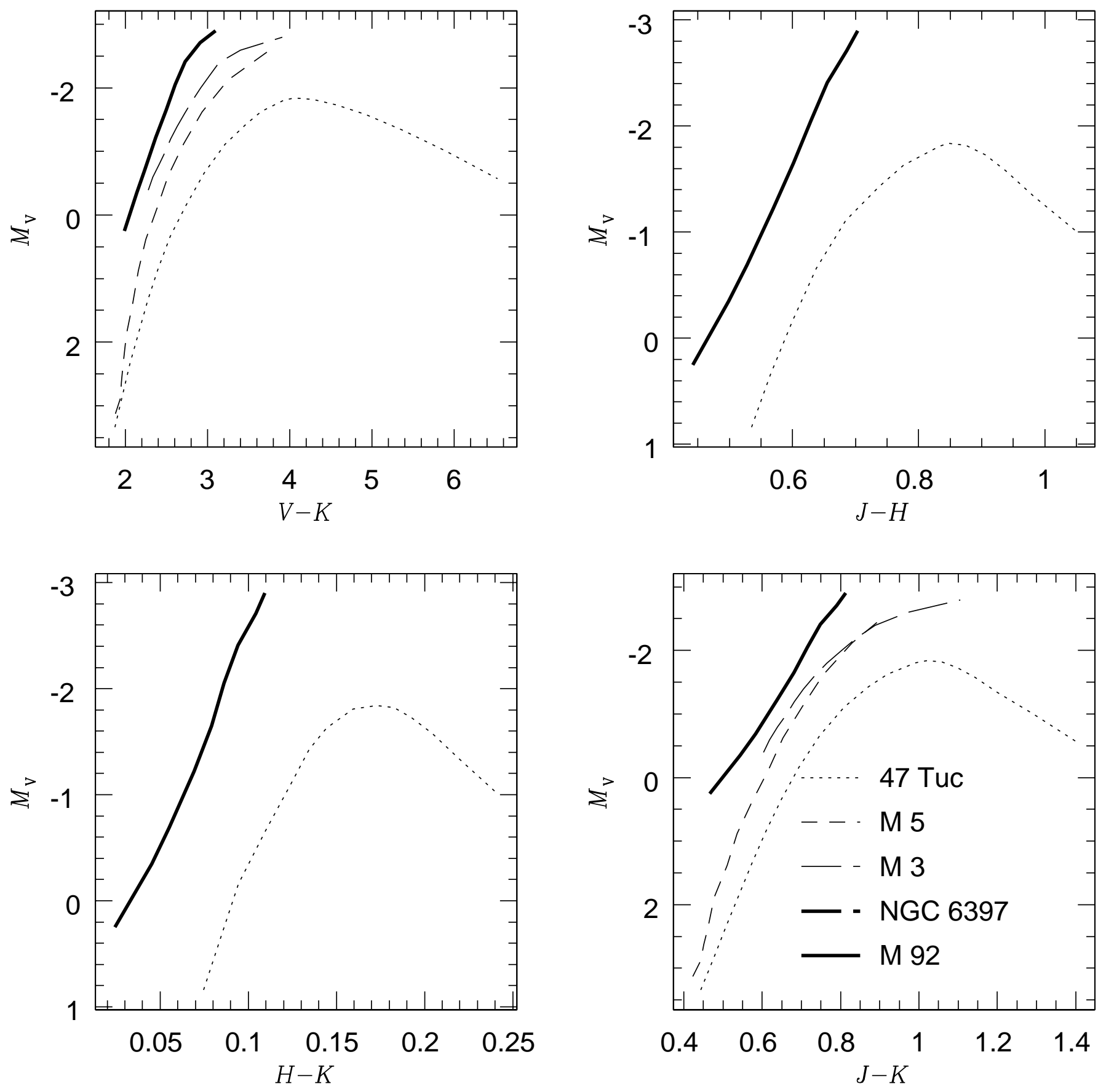

Fig. 2. Empirical infrared fiducial lines in the CMD of the globular clusters 47 Tuc, M 5, M 3, NGC 6397 and M 92 . For $V-K$ and $J-K$, no NGC 6397 data were available, and for $J-H$ and $H-K$, only 47 Tuc and M 92 data were found.

calibration files, we had no choice but to adopt the $T_{\text {eff }}-$ $K-L$ relation directly from the BaSeL 2.2 library.

This set (i.e. metallicity-dependent colour-temperature $-\log g$ ) was complemented with the solar relations synthesised by Lejeune et al. to calibrate the BaSeL 2.2 library (see Paper II). The so-derived calibration files (two for each metallicity $([\mathrm{Fe} / \mathrm{H}]=0,-0.5,-1,-1.5,-2)$, one for (red) giants and one for dwarfs) are available by public ftp from the university of Basel.

\section{Metallicity-dependent library calibration, the BaSeL 3.1 "WLBC 99" library}

These calibration files were then used to colour-calibrate the hybrid library of theoretical SEDs (consisting of the Kurucz-, the Allard and Hauschildt-, and the Scholzmodels) (Kurucz 1995; Allard \& Hauschildt 1995; Scholz 1997) using the (modified) algorithm developed by Cuisinier et al. (1997, see Paper I). For each temperature, 
Table 1. Sources of photometric data of globular clusters.

\begin{tabular}{|c|c|c|c|}
\hline cluster, $[\mathrm{Fe} / \mathrm{H}],(m-M)_{0}, E_{B-V}$ & colour bands & source & range (in $m_{V}$ ) \\
\hline \multirow[t]{8}{*}{47 Tuc (NGC 104), $-0.70,13.14,0.04$} & $B-V$ & Kaluzny et al. (1998) & $\lesssim 19^{\mathrm{m}}$ \\
\hline & $B-V$ & Hesser et al. (1987) & $\sim 24^{\mathrm{m}}-\sim 19^{\mathrm{m}}$ \\
\hline & $V-I$ & Bica et al. (1994) & $\lesssim 13.5^{\mathrm{m}}$ \\
\hline & $V-I$ & Kaluzny et al. (1998) & $\sim 21^{\mathrm{m}}-\sim 13.5^{\mathrm{m}}$ \\
\hline & $U-B$ & Mermilliod $(1998)^{a}$ & $\sim 16^{\mathrm{m}}-\sim 12^{\mathrm{m}}$ \\
\hline & $V-R^{b}$ & Cathey (1974) & $\sim 16^{\mathrm{m}}-\sim 12^{\mathrm{m}}$ \\
\hline & $V J K$ & Montegriffo et al. (1995) & $\lesssim 17^{\mathrm{m}}$ \\
\hline & $H-K$ & Frogel et al. (1981) & $\lesssim 14.5^{\mathrm{m}}$ \\
\hline \multirow[t]{6}{*}{ M 5 (NGC 5904), -1.11, 14.32, 0.03} & $B-V$ & Sandquist et al. (1996) & $\widetilde{\lesssim} 22^{\mathrm{m}}$ \\
\hline & $B-V$ & Richer \& Fahlman (1987) & $\sim 24^{\mathrm{m}}-\sim 22^{\mathrm{m}}$ \\
\hline & $V-I$ & Sandquist et al. (1996) & $\lesssim 22^{\mathrm{m}}$ \\
\hline & $U-B$ & $\begin{array}{l}\text { Drissen \& Shara (1998), } \\
\text { von Braun et al. (1998) }\end{array}$ & $\widetilde{\lesssim} 19.5^{\mathrm{m}}$ \\
\hline & $V-R$ & von Braun et al. (1998) & $\lesssim 17.5^{\mathrm{m}}$ \\
\hline & $J-K$ & Frogel et al. (1983) & $\lesssim 17.5^{\mathrm{m}}$ \\
\hline \multirow[t]{9}{*}{ M 3 (NGC 5272), -1.34, 15.01, 0.01} & $B-V$ & Ferraro et al. (1997) & $\widetilde{\lesssim}_{22} 2^{\mathrm{m}}$ \\
\hline & $V-I$ & Ferraro et al. (1997) & $\lesssim 16^{\mathrm{m}}$ \\
\hline & $V-I$ & Johnson \& Bolte (1998) & $\sim 23^{\mathrm{m}}-\sim 16^{\mathrm{m}}$ \\
\hline & $V-I$ & Marconi et al. (1998) & $\sim 27^{\mathrm{m}}-\sim 23^{\mathrm{m}}$ \\
\hline & $U-B$ & Mermilliod $(1998)^{c}$ & $\lesssim 21.5^{\mathrm{m}}$ \\
\hline & $V-R^{b}$ & Arribas \& & $\lesssim 14.5^{\mathrm{m}}$ \\
\hline & & Martínez-Roger (1987) & \\
\hline & $V-K$ & Frogel et al. (1981) & $\lesssim 15^{\mathrm{m}}$ \\
\hline & $J-K$ & Lee et al. (1996) & $\underset{\sim}{\lesssim} 15^{\mathrm{m}}$ \\
\hline \multirow[t]{8}{*}{ NGC $6397,-1.82,11.75,0.18$} & $B-V$ & Kaluzny (1997) & $\lesssim 18.5^{\mathrm{m}}$ \\
\hline & $B-V$ & Alcaino et al. (1997) & $\sim 21.5^{\mathrm{m}}-\sim 18.5^{\mathrm{m}}$ \\
\hline & $U-B$ & Alcaino et al. (1997) & $\sim 20^{\mathrm{m}}-\sim 16.5^{\mathrm{m}}$ \\
\hline & $U-B$ & Mermilliod $(1998)^{d}$ & $\lesssim 16.5^{\mathrm{m}}$ \\
\hline & $V-I$ & Alcaino et al. (1997) & $\lesssim 12.5^{\mathrm{m}}$ \\
\hline & $V-I$ & Alcaino et al. (1997) & $\sim 16.5^{\mathrm{m}}-\sim 12.5^{\mathrm{m}}$ \\
\hline & $V-I$ & King et al. (1998) & $\sim 27^{\mathrm{m}}-\sim 16.5^{\mathrm{m}}$ \\
\hline & $R-I$ & Alcaino \& Liller (1985) & $\lesssim 16.25^{\mathrm{m}}$ \\
\hline \multirow[t]{8}{*}{ M 92 (NGC 6341), -2.16, 14.53, 0.02} & $B-V$ & Sandage (1970) & $\lesssim 21.5^{\mathrm{m}}$ \\
\hline & $B-V$ & Stetson \& Harris (1988) & $\sim 24^{\mathrm{m}}-\sim 21.5^{\mathrm{m}}$ \\
\hline & $V-I$ & von Braun et al. (1998) & $\lesssim 14^{\mathrm{m}}$ \\
\hline & $V-I$ & Johnson \& Bolte (1998) & $\sim 20^{\mathrm{m}}-\sim 14^{\mathrm{m}}$ \\
\hline & $V-I$ & Piotto et al. (1997) & $\sim 28^{\mathrm{m}}-\sim 20^{\mathrm{m}}$ \\
\hline & $U-B$ & Mermilliod $(1998)^{e}$ & $\lesssim 20.5^{\mathrm{m}}$ \\
\hline & $V-K$ & Frogel et al. (1981) & $\lesssim 15^{\mathrm{m}}$ \\
\hline & $J H K^{f}$ & Cohen et al. (1978) & $\lesssim 15^{\mathrm{m}}$ \\
\hline
\end{tabular}

${ }^{a}$ Alcaino \& Liller (1985), Evans (1983), Norris \& Freeman (1982), Lee (1977), Demarque \& McClure (1977), Hesser \& Hartwick (1977), Cannon (1974), Menzies (1973), Eggen (1972).

${ }^{b}$ Transformed to Johnson-Cousins using Bessell (1983).

${ }^{c}$ Johnson \& Sandage (1956), Sandage $(1969,1970)$.

${ }^{d}$ Woolley et al. (1961), Newell et al. (1969), Cannon (1974), Alcaino (1977), van den Bergh (1988).

e Sandage \& Walker (1966), Sandage (1969, 1970) Eggen (1972).

${ }^{f}$ Transformed to Johnson using Bessell \& Brett (1988).

it calculates a correction function which must be multiplied with the theoretical spectrum corresponding to the parameters given in the calibration file for that temperature, such that the corrected spectra reproduce the colours given in the calibration file. This correction function being smooth, it leaves line strength indices untouched and only changes the continuum. This correction function is then used to correct all model spectra of this temperature, whereby the original differential properties with $\log g$ and
$[\mathrm{Fe} / \mathrm{H}]$ are usually conserved, and hence, continue to be well represented by the models. In the end, all SEDs are rescaled to make the bolometric fluxes match the temperatures (for a more precise description, see Paper I). Thus, the new feature of the calibration algorithm is to waive this differential approach in favour of calculating a separate correction function each temperature and for each level of $[\mathrm{Fe} / \mathrm{H}]$, the differential properties with $\log g$ of the models are preserved, however. On top of that, the 
Table 2. Temperature ranges of empirical colour- $T_{\text {eff }}$ relations.

\begin{tabular}{lclc}
\hline Luminosity class & {$[\mathrm{Fe} / \mathrm{H}]$} & Colours & temperature range $[\mathrm{K}]$ \\
\hline \multirow{2}{*}{ giants } & -0.5 & UBVRIJHK ${ }^{a}$ & $3350-6000$ \\
& -1.0 & UBVRIJHK & $3750-6000$ \\
& -1.5 & UBVRIJHK & $3750-6000$ \\
& -2.0 & UBVRIJHK & $4000-6000$ \\
\multirow{2}{*}{ dwarfs } & all & $K-L$ & - \\
& all & $U B V I^{b}$ & $4500-10000$ \\
\hline
\end{tabular}

\footnotetext{
${ }^{a} U B V R I J H K: U-B, B-V, V-I, R-I, V-K, J-H, H-K, J-K$.

${ }^{b} U B V I: U-B, B-V, V-I$.

${ }^{c} R J H K L: R-I, V-K, J-H, H-K, J-K, K-L$.
}

calibration programs were modified to include a smooth transition between the giant-calibrated and the dwarfcalibrated range.

In this way, we created a metallicity-calibrated library of synthetic stellar SEDs, designed to reproduce observed colour-colour - and colour-temperature relations, which (for historical reasons) will from now on be called the BaSeL 3.1 "WLBC 99" library.

As a first test of the new SED library, it was used to derive spectra and colours from the Yale and the Padova isochrones for the grid metallicities and for age 0 and 14 Gyr and also for the observed cluster metallicities at age 14 Gyr. As can be seen from Figs. 3 and 4 - where the input (calibration) relations (solid) are shown against the output (model) relations (dashed) and the BaSeL 2.2 semi-empirical relations (dotted) - the used colourtemperature relations are well-reproduced. This mainly confirms the functionality of the calibration algorithm, but also suggests that the colour-temperature relations of the models can be trusted. Because these relations have been calibrated in a metallicity-dependent way, the present results show improvements over earlier results obtained from the semi-empirical (BaSeL 2.2) library calibration in $U-B, J-H, H-K$ and $J-K$, especially at low temperatures. They also appear smoother than their predecessors. Below the lower temperature limits of the calibration relations specified in Table 2, they still show the same discontinuities as the BaSeL 2.2 models, due to lack of data, but for population synthesis, this shouldn't pose major problems, as stars of these low temperatures either don't show up at all (giants), or contribute only negligibly to the integrated light of a population (dwarfs).

The second test unfortunately yields much less satisfying results. $M_{V}-U B V R I J H K$ CMDs were produced using Yale and Padova isochrones for the observed metallicities of the calibrating globulars and typical cluster ages (e.g. 10, 12, 12, 14 and 16 Gyr for 47 Tuc, M 5, M 3, NGC 6397, and M 92 respectively), in order to reproduce their CMDs shown in Figs. 1 and 2. Contrary to expectations, these isochrones do not reproduce the cluster CMDs better than the BaSeL 2.2 semi-empirical models. The RGBs come out too steep and in certain colours $(U-B$, $B-V, V-I)$ too red for high metallicities, and too blue in all colours for low values of $[\mathrm{Fe} / \mathrm{H}]$ (see Figs. 5 and 6 ).

Obviously, it is impossible at the present epoch to establish a unique calibration of $U B V R I J H K L$ colours in terms of stellar metallicity that reproduces both empirical colour-temperature relations of stars and the CMDs of stellar populations (using theoretical isochrones). Whether these discrepancies are due to the used colourtemperature relations, or to the isochrones remains to be investigated, but recent indications point towards shortcomings in the isochrones, especially in the convection treatment (Salaris 2001).

Figures 3, 4, 5, and 6 are only shown for Padova isochrones, but the results also hold for Yale isochrones, and the corresponding figures look very similar. The SED library is available by public ftp from the university of Basel as the BaSeL 3.1 "WLBC 99" version. Because of its good agreement for high metallicities, the (unmodified) $[\mathrm{Fe} / \mathrm{H}]=+0.5$ file from the BaSeL 2.2 library has been added to extend the metallicity range.

\section{Pragmatic solution: Application-oriented library calibration, the BaSeL 3.1 "Padova 2000" library}

As obviously both the colour-temperature relations of stars and the CMDs of stellar populations (using existing theoretical isochrones) cannot be matched at the same time, we decided to also produce a library that is able to reproduce the CMDs of globulars well, because such a library should at least yield reliable integrated colours for stellar populations and hopefully reliable integrated spectra. Another motivation was the fact that according to some authors (Brocato et al. 2000), colour-temperature relations, in particular for cool stars, aren't that wellknown anyway. These authors even consider them a free parameter. 

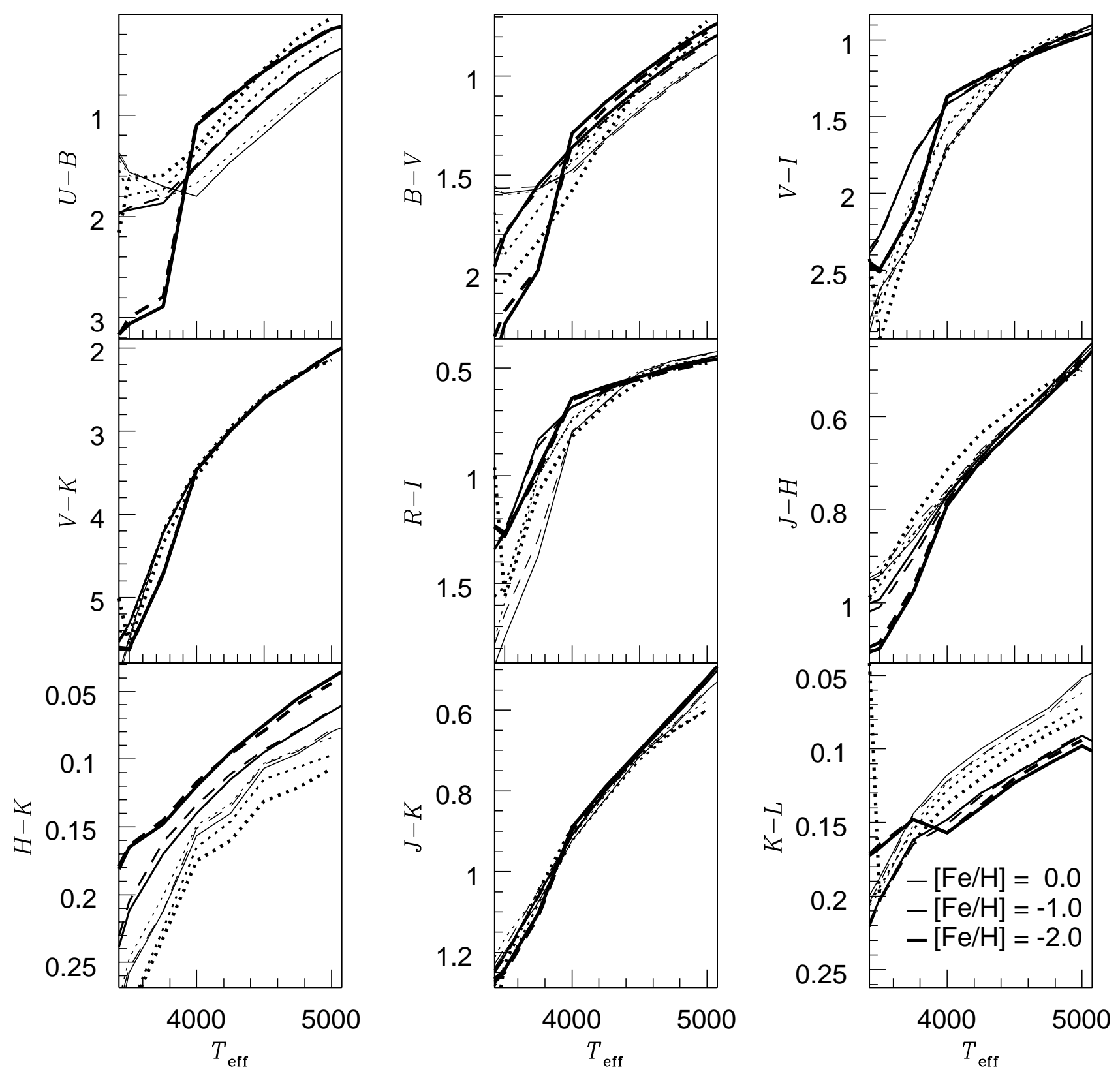

Fig. 3. Empirical colour-temperature relations for giants of three grid metallicities (solid lines, increasing line width means decreasing metallicity) versus the relations from the BaSeL 3.1 "WLBC 99" models (dashed) resp. the BaSeL 2.2 semi-empirical models (dotted) for the same parameters.

As a theoretical isochrone library, which, combined with our to-be-produced SED library, should reproduce the CMDs of our calibration globular clusters, we chose the Padova 2000 isochrones (Girardi et al. 2000), because it is widely in use nowadays.

We produced this library in an iterative process that is based on the fact (observed by us), that the $M_{V}$ magnitude, derived for a set of parameters $[\mathrm{Fe} / \mathrm{H}], T_{\text {eff }}$, and $\log g$ from a stellar library, is practically independent of the choice of the spectral library. This is easily explained by the fact that $M_{\mathrm{bol}}$ depends only on the stellar parameters (thus is independent of the choice of library), so the difference in $M_{V}$ stems only from the difference in the bolometric correction $B C(V)$ derived from the different libraries, which is negligible on the scale of absolute magnitudes. This library independence of $M_{V}$ can now be used to assign the colours of one's choice to a certain $M_{V}$ value, by assigning them to the $T_{\text {eff }}$ and $\log g$ values that will reproduce this $M_{V}$ value in the to-be-calibrated library, which one knows already from an existing library. This way one can in principle shape a synthetical colour-magnitude diagram in the way one desires ${ }^{2}$.

The iterative process employed was the following:

1. New calibration files were created from the $T_{\text {eff }}-\log g$ relations of the Padova 2000 isochrones for $0 \mathrm{Gyr}$ for

${ }^{2}$ Note that this procedure implies giving up consistency with empirically established $T_{\text {eff }}$-colour relations. 

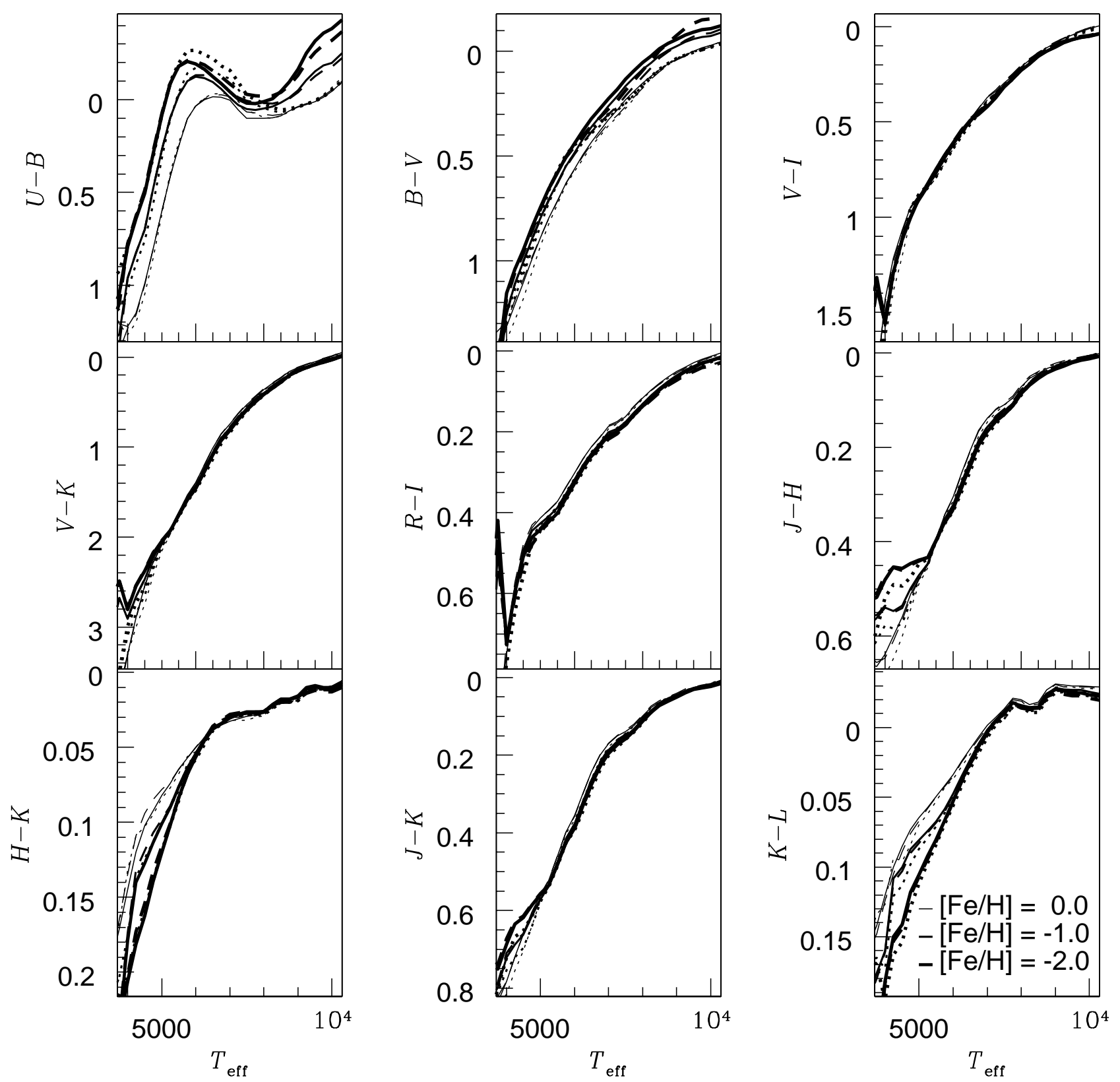

Fig. 4. Empirical colour-temperature relations for dwarfs of three grid metallicities (solid lines, increasing line width means decreasing metallicity) versus the relations from the BaSeL 3.1 "WLBC 99" models (dashed) resp. the BaSeL 2.2 semi-empirical models (dotted) for the same parameters.

dwarfs (the age at which the main sequence is still complete) and 14 Gyr for giants (a typical globular cluster age), and colour-temperature relations derived via the colour $-M_{V}$ relations from the globular cluster fiducials presented in Sect. 2 and the $M_{V}-T_{\text {eff }}$ relations derived with the before-mentioned Padova 2000 isochrones from the previous library (in the first step, the "previous library" was the "WLBC 99" library, and in the following steps, it was the library produced in the previous step). For solar metallicity, the calibration file was kept the same as for the "WLBC 99" library (thus also the same as for the BaSeL 2.2 library), with the small difference, that the $T_{\text {eff }}-\log g$ relation was taken from the Padova 2000 isochrones for consistency;

2. These calibration files were used to calibrate a new library;

3. Steps 1 and 2 were repeated until there was no significant difference anymore between subsequent libraries.

After four iteration steps this was the case. (The calibration files used for the final iteration are also available by ftp from Basel university, although they are probably of little use to the user.)

As can be seen from Figs. 7 and 8, the above procedure indeed provides the expected improvements. They show 


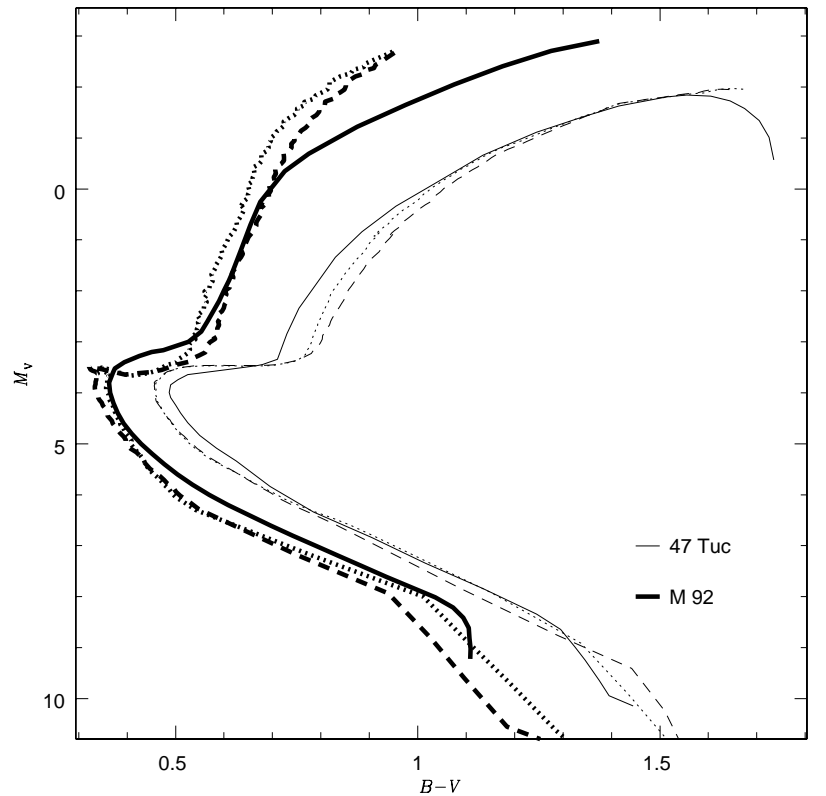

Fig. 5. Empirical $(B-V)-M_{V}$ CMD of the globular clusters 47 Tuc and M92 (solid). Overlaid are the CMDs created by combining the Padova 10 Gyr isochrone for $[\mathrm{Fe} / \mathrm{H}]=$ -0.70 and the $16 \mathrm{Gyr}$ isochrone for $[\mathrm{Fe} / \mathrm{H}]=-2.16$ with the BaSeL 3.1 "WLBC 99" library (dashed) resp. with the BaSeL 2.2 semi-empirical library (dotted).

the globular cluster CMDs of Figs. 1 and 2 compared to the Padova 2000 isochrones of the same (cluster) metallicities and the above-mentioned ages evaluated with the new BaSeL 3.1 "Padova 2000" library.

The (metallicity-dependent) shapes and locations of the RGB and the main sequence are well-reproduced for the entire range from $[\mathrm{Fe} / \mathrm{H}]=-2.16$ to -0.70 , apart from the RGB tip of 47 Tuc, of which the "bend-down" in $V$ proved virtually impossible to reproduce (for solar $[\mathrm{Fe} / \mathrm{H}]$, the quality of the library has already been confirmed extensively, as in its present form, it is almost identical with the Lejeune et al. (1998) BaSeL 2.2 library, which is widely used). At a given metallicity, the CMDs of individual clusters, however, can still differ by as much as $0.1^{\mathrm{m}}$ in colour, due to the above-mentioned large intrinsic cluster-by-cluster scatter in the observed CMDs. From the generally good overall agreement, it can be concluded that this new library should be useful for population synthesis, if combined with Padova 2000 isochrones or tracks. It can be retrieved by ftp from Basel university as the BaSeL 3.1 "Padova 2000" library. Here too, the unmodified $[\mathrm{Fe} / \mathrm{H}]=+0.5$ file from the BaSeL 2.2 library was added.

The good agreement in the CMDs comes at the price of rather unusual colour temperature - relations. Figure 9 shows the empirical $T_{\text {eff }}-V-K$ relation from Ridgway et al. (1980) and empirical $T_{\text {eff }}-\log g$ relations for red giants from Cohen, Frogel and Persson (Cohen et al. 1978; Frogel et al. 1981, 1983, [Fe/H]-dependent) and dwarfs from Angelov (1996, [Fe/H]-independent) for three metallicities from the grid (solid lines) versus the BaSeL 3.1

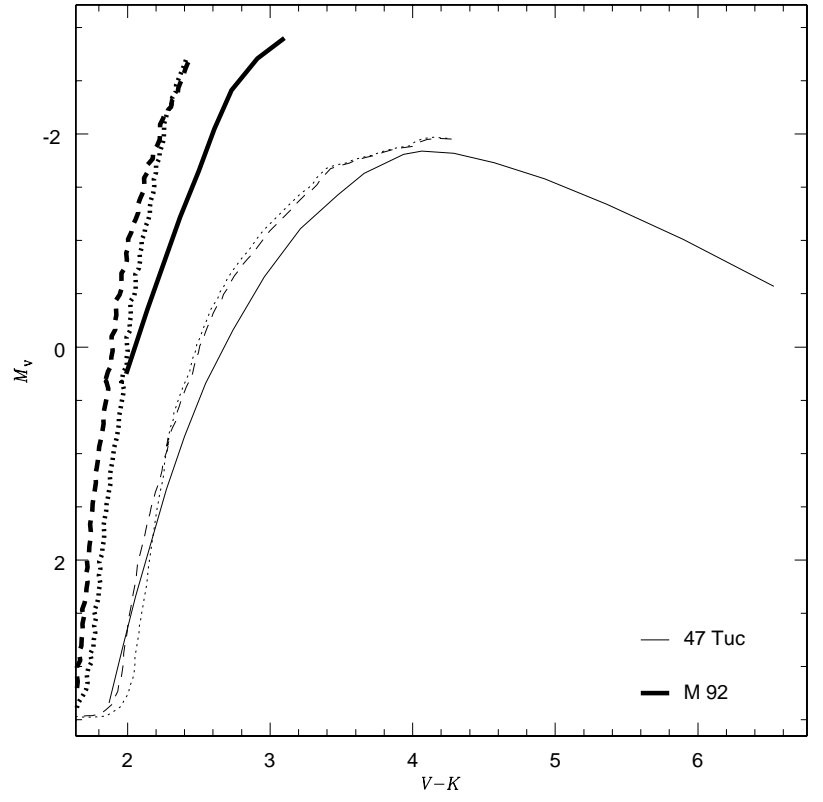

Fig. 6. Empirical $(V-K)-M_{V}$ CMD of the globular clusters 47 Tuc and M92 (solid). Overlaid are the CMDs created by combining the Padova $10 \mathrm{Gyr}$ isochrone for $[\mathrm{Fe} / \mathrm{H}]=$ -0.70 and the 16 Gyr isochrone for $[\mathrm{Fe} / \mathrm{H}]=-2.16$ with the BaSeL 3.1 "WLBC 99" library (dashed) resp. with the BaSeL 2.2 semi-empirical library (dotted).

"Padova 2000" (dashed) and the BaSeL 2.2 semi-empirical (dotted) relations for the same parameters. For giants, the disagreement reaches up to $500 \mathrm{~K}$ or $1^{\mathrm{m}}$ in $V-K$ (for colours with a smaller baseline, this error can be scaled down accordingly); for the lowest metallicities and temperatures $\left([\mathrm{Fe} / \mathrm{H}] \leq-1.5\right.$ and $\left.T_{\text {eff }} \leq 4000 \mathrm{~K}\right)$ it can even reach $800 \mathrm{~K}$, but fortunately, these stars don't show up in the Padova 2000 isochrones. For dwarfs, these problems aren't as bad as for giants, but it is clear that temperatures derived from the BaSeL 3.1 "Padova 2000" models should be treated with scepticism. On the positive side, most of the discontinuities have disappeared in the colour temperature - relations.

In a last test, the effect of the metallicity-calibration on the integrated spectra of synthetic single-burst stellar populations was investigated. Synthetic populations were created in order to compare their integrated spectra with empirical template globular cluster integrated energy distributions from Bica et al. (1996, private communication). The synthetic populations were created using the GISSEL (Galaxy Isochrone Synthesis Spectral Evolution Library) software by G. Bruzual A. and S. Charlot (2000, for a short description, see Charlot \& Bruzual 1991; Bruzual \& Charlot 1993) for the template metallicities $([\mathrm{Fe} / \mathrm{H}]=0.25,0.00,-0.50,-1.00,-1.50,-2.00)$, and the same ages as Lejeune used in his thesis (1997) for comparison of the BaSeL 2.2 semi-empirical library with the same template spectra $(12,14,14,18,20$ Gyr). Both the BaSeL 2.2 semi-empirical - and the BaSeL 3.1 "Padova 2000" SED libraries were used, along with a Salpeter IMF (mass range: $0.1-50 M_{\odot}$ ) and the Padova 2000 tracks 

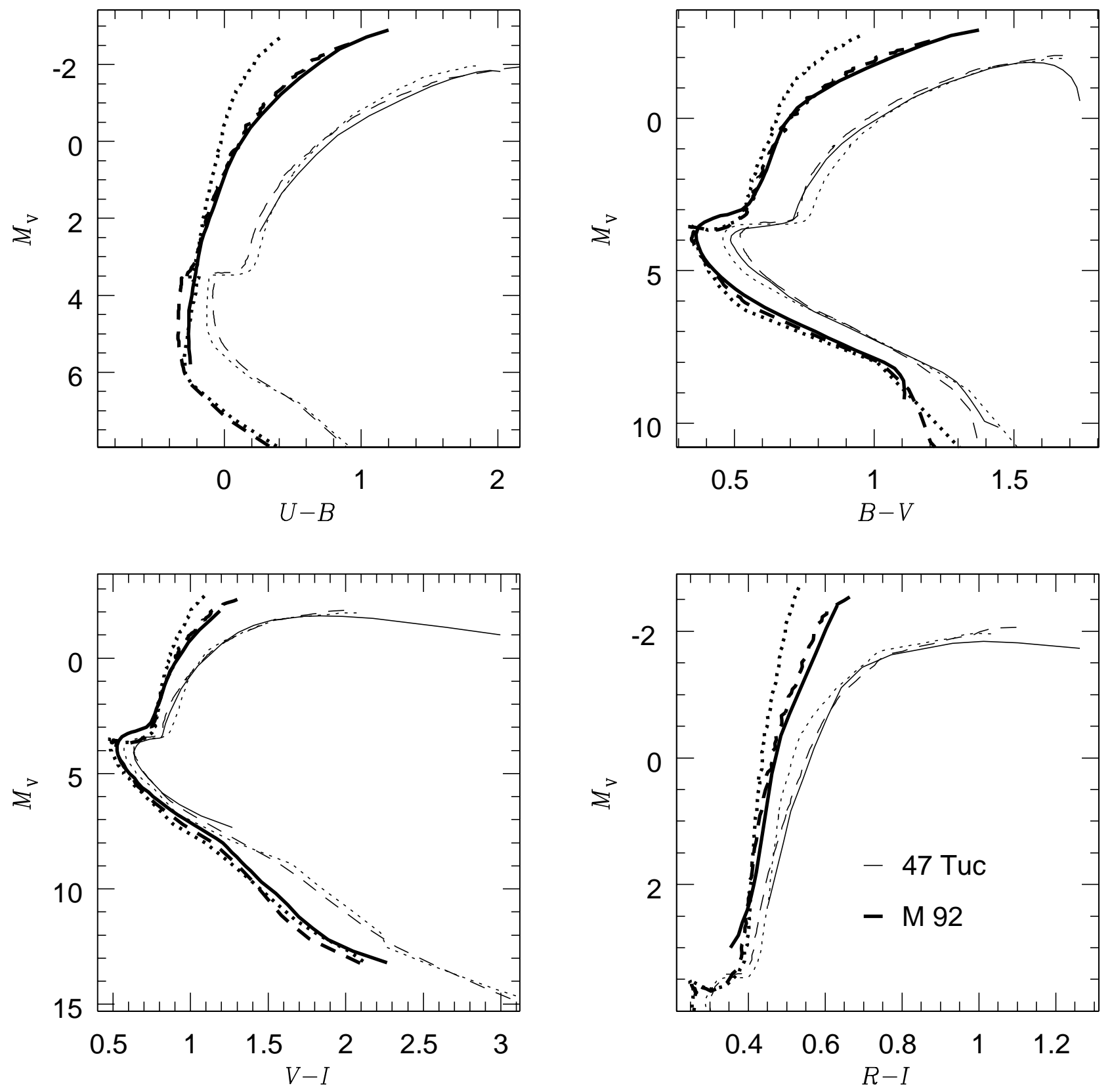

Fig. 7. Empirical ( $U B V R I)$ CMDs of the globular clusters 47 Tuc and M92 (solid). Overlaid are the CMDs created by combining the Padova $10 \mathrm{Gyr}$ isochrone for $[\mathrm{Fe} / \mathrm{H}]=-0.70$ and the $16 \mathrm{Gyr}$ isochrone for $[\mathrm{Fe} / \mathrm{H}]=-2.16$ with the BaSeL 3.1 "Padova 2000" library (dashed) resp. with the BaSeL 2.2 semi-empirical library (dotted).

(except for $[\mathrm{Fe} / \mathrm{H}]=-2.0$, where the Padova 1995 isochrone (Fagotto et al. 1994; Girardi et al. 1996) was used, because the corresponding Padova 2000 isochrone wasn't implemented in the evolutionary synthesis program). The resulting integrated spectra are compared in Fig. 10, where the solid lines stand for the empirical template spectra, the dotted lines for the BaSeL 2.2 semiempirical library and the dashed lines for the metallicitycalibrated library. Residuals in the sense BaSeL $2.2-$ empirical spectra (dotted), resp. BaSeL 3.1 - empirical spectra (solid) are shown in the same figure. To guide the eye, the zero-line is also shown in solid. Synthetic (UBVRIJHKLM) colours and magnitudes from the empirical - and the synthetical (BaSeL 2.2 "Padova 2000") spectra are given in Tables 3 and 4 , and the differences between these colours in Table 5 (due to the limited wavelength range of the Bica et al. spectra, only $U B V R I$ colours can be given for the empirical spectra). As expected, the differences between the two libraries are negligible for high $[\mathrm{Fe} / \mathrm{H}]$. They are hardly visible in Fig. 10, and in the colours, they are of the order of $0.001^{\mathrm{m}}$. For low metallicities $([\mathrm{Fe} / \mathrm{H}]=-1.65)$, the metallicity-calibration seems to add flux in the red $(R, I)$ and to reduce the flux in the visible a bit, but clearly, the dramatic differences in stellar colour-temperature relations between the two libraries have only a minor effect 

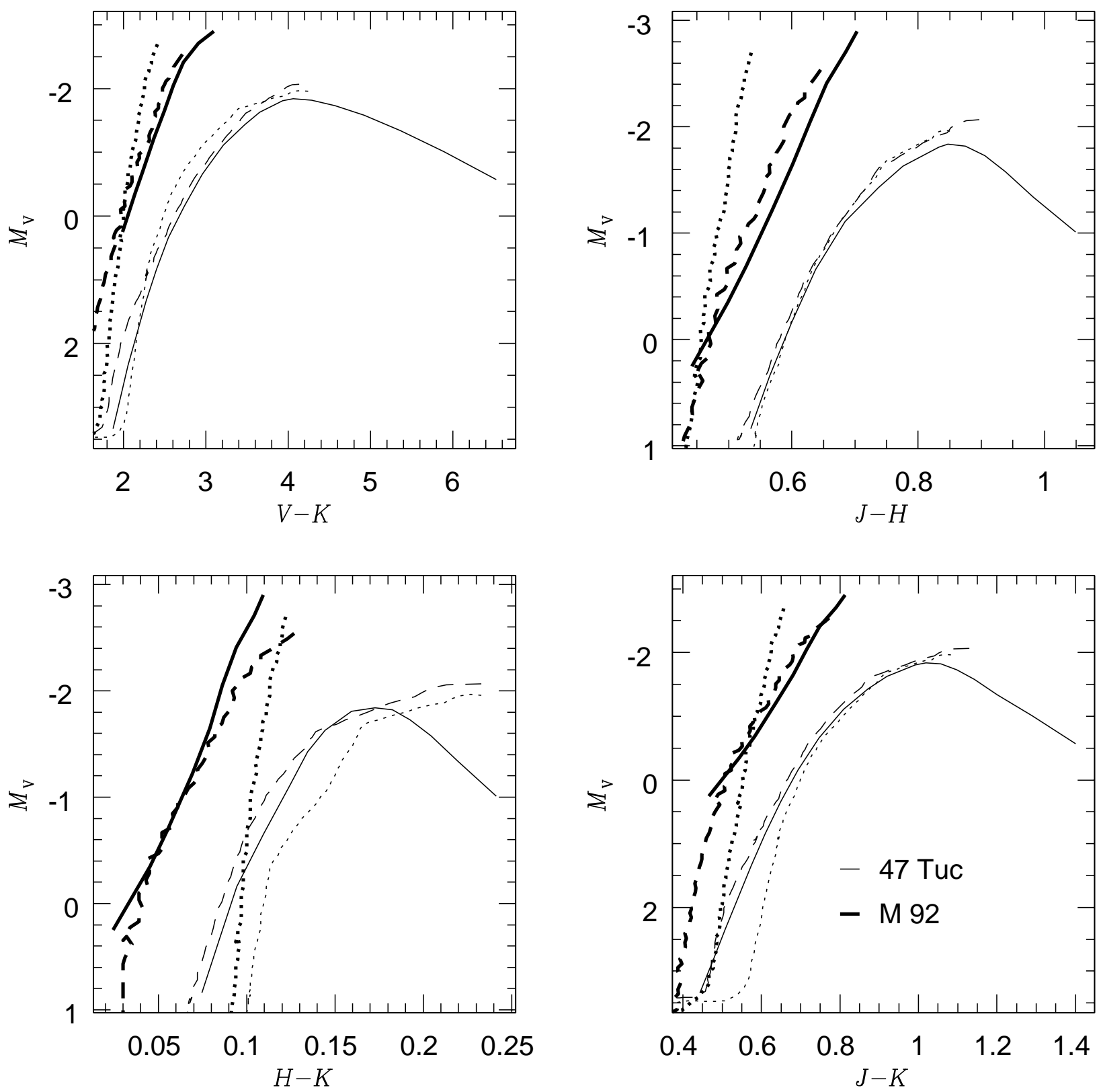

Fig. 8. As in Fig. 7, but for infrared colours. The steepening in $J-H, H-K$ and $J-K$ of the M 92 RGB above $M_{V}=\sim-1$ was deliberately not reproduced, as it's probably not a common RGB feature (maybe the tip of this fiducial was actually produced by asymptotic giant branch stars).

Table 3. Colours for template globular cluster integrated energy distributions from Bica et al. (1996, private communication).

\begin{tabular}{lrrrrr}
\hline template & {$[\mathrm{Fe} / \mathrm{H}]$} & $U-B$ & $B-V$ & $V-I$ & $R-I$ \\
\hline$G 177$ & 0.25 & - & - & 1.235 & 0.653 \\
$G 1-M 31 c l$ & 0.00 & - & 0.993 & 1.238 & 0.663 \\
$G 2 b-M 31 c l$ & -0.50 & 0.296 & 0.869 & 1.117 & 0.585 \\
$G 3 r$ & -1.00 & 0.093 & 0.739 & 1.044 & 0.549 \\
$G 4 b$ & -1.50 & 0.041 & 0.676 & 1.032 & 0.551 \\
$G 5$ & -2.00 & -0.001 & 0.639 & 0.948 & 0.507 \\
\hline
\end{tabular}

on the spectral properties of entire (synthetic) populations. The main improvements can be seen in the visible (the spectral shape in this region is matched perfectly for all metallicities), whereas in the ultraviolet one can still see some strong deviations. However, the differences between empirical integrated colours and the ones of the synthetic population only amount to a few hundredths of a magnitude (see Table 5), confirming that the BaSeL 3.1 "Padova 2000" SED library should be a useful tool for evolutionary synthesis, if used in combination with the Padova 2000 tracks/isochrones.

\section{Conclusions and future work}

1. We have constructed two comprehensive libraries of theoretical stellar energy distributions combining the Kurucz -, the Allard and Hauschildt -, and the 

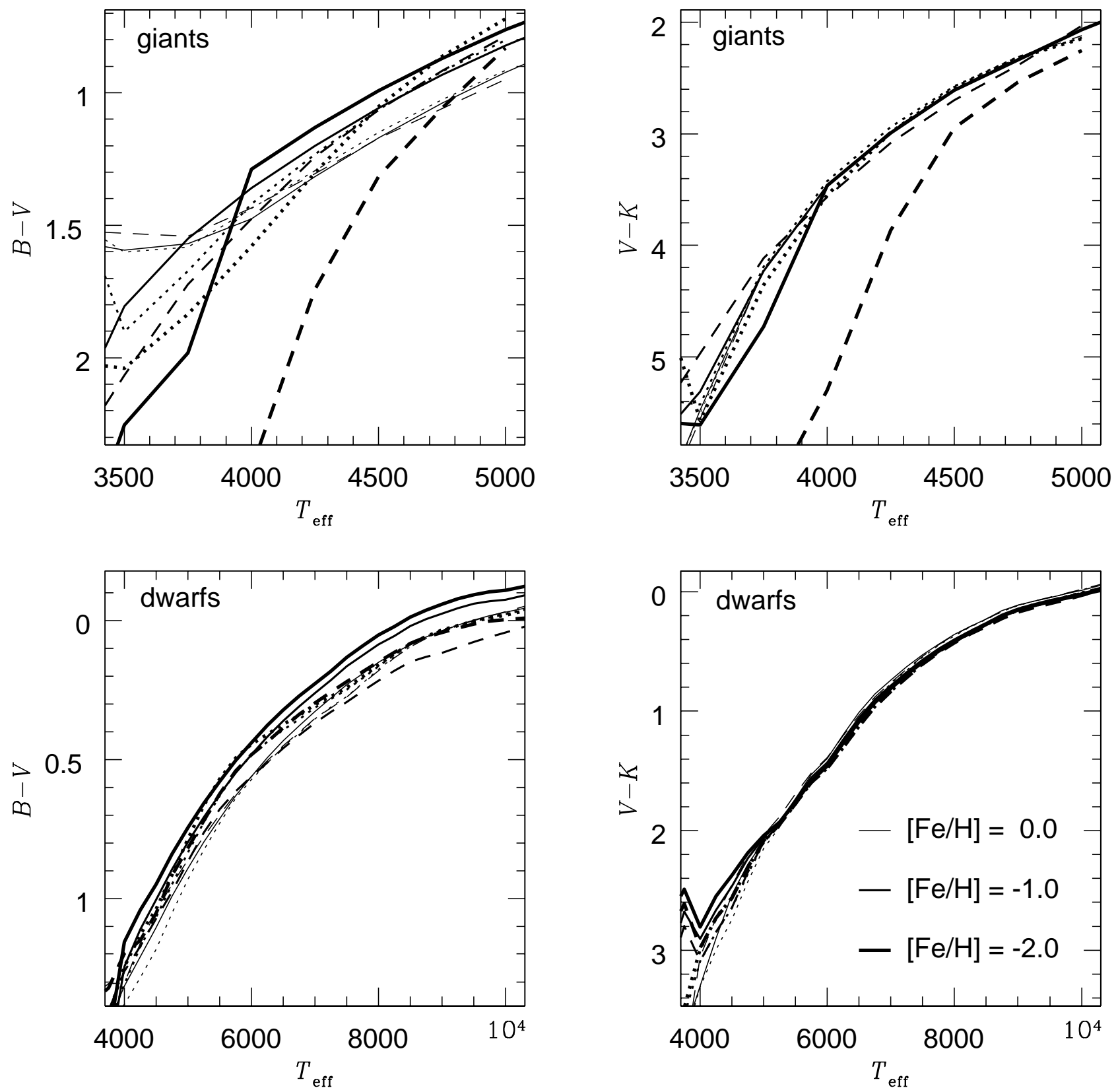

Fig. 9. Empirical colour-temperature relations for giants and dwarfs of three grid metallicities (solid lines, increasing line width means decreasing metallicity) versus the relations from the BaSeL 3.1 "Padova 2000" models (dashed) resp. the BaSeL 2.2 semi-empirical models (dotted) for the same parameters.

Scholz-models (Kurucz 1995; Allard \& Hauschildt 1995; Scholz 1997). They provide synthetic stellar spectra with useful resolution on a homogeneous wavelength grid, from $9.1 \mathrm{~nm}$ to $160 \mu \mathrm{m}$, over large ranges of fundamental parameters. In addition to the preceding version, the BaSeL 2.2 semi-empirical library (see Papers I and II), the present libraries have been (UBVRIJHKL) colour-calibrated independently at all levels of metallicity, using Galactic globular cluster photometric data, to overcome the weaknesses of the BaSeL 2.2 library at low $[\mathrm{Fe} / \mathrm{H}]$.

2. It proved impossible to calibrate the library in such a way that both the empirical colour-temperature relations (taken from Ridgway et al. 1980) and the CMDs of the calibrating clusters (using Yale or Padova 2000 isochrones) could be matched at the same time. Whether this is due to the isochrones or to irrealistic colour-temperature relations still remains to be investigated. Of great help would also be a more complete (colour) data basis. For this reason, we created two metallicity-calibrated libraries, the BaSeL 3.1 "WLBC 99" library, which is able to reproduce empirical colour-temperature relations and should therefore be a useful tool for investigations on a stellar level, and the BaSeL 3.1 "Padova 2000" library which, in combination with the Padova 2000 isochrones, successfully 


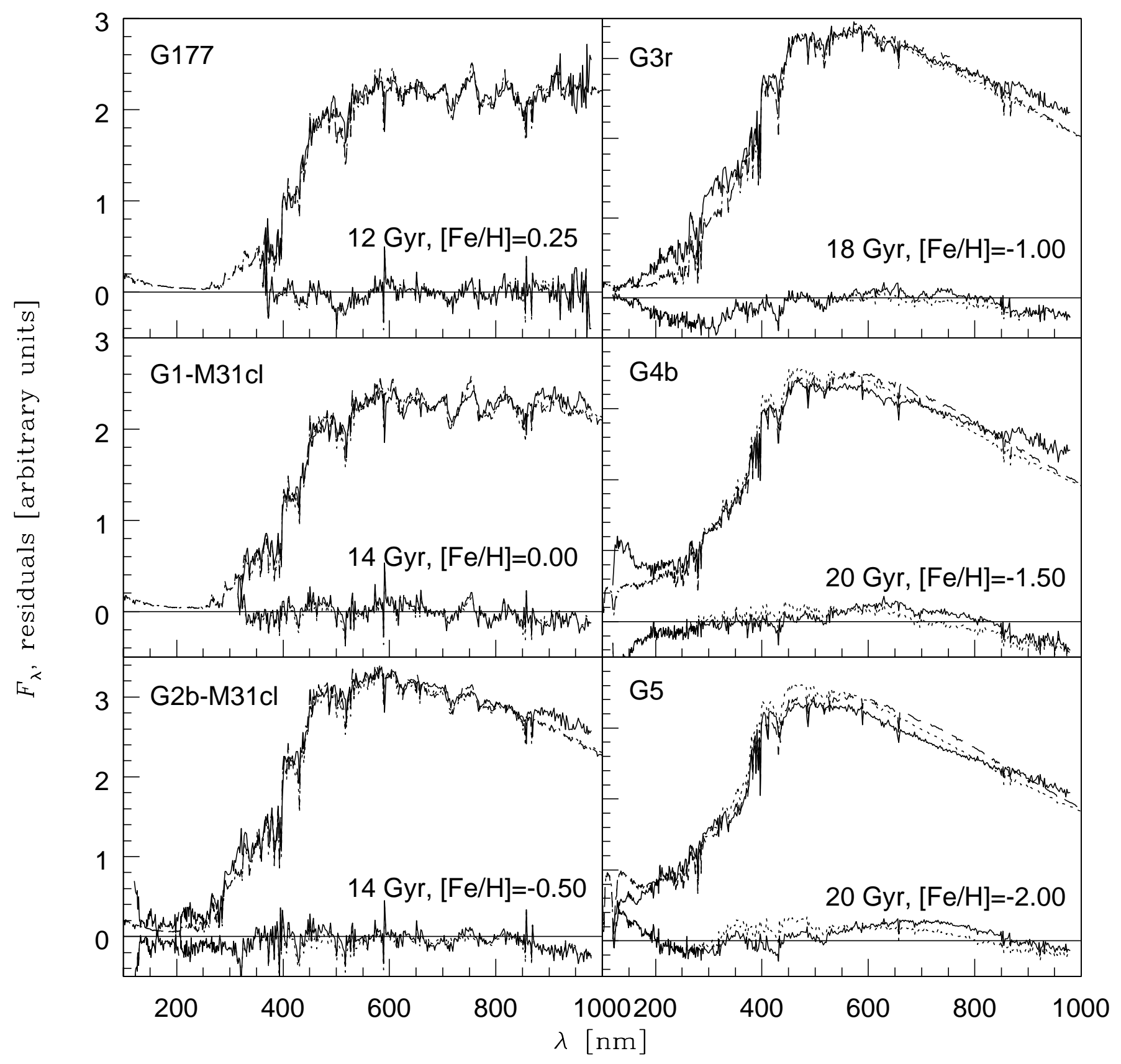

Fig. 10. Template globular cluster integrated energy distributions from Bica et al. (1996, private communication) (solid lines) vs. integrated spectra of synthetic single burst stellar populations for the given ages and metallicities using the BaSeL 2.2 semi-empirical SED library (dotted) or the BaSeL 3.1 "Padova 2000" SED library (dashed). At the bottom of the figures, the residuals are shown, in the sense BaSeL 2.2 - empirical spectra (dotted), or BaSeL 3.1 - empirical spectra (solid). The zero-line is also shown in solid.

reproduces the CMDs (i.e. the location and shape of the different branches) of Galactic globular clusters, and which should therefore be a particularly suitable tool for spectral evolutionary synthesis studies of stellar systems and other synthetic photometry applications, especially if used in combination with the Padova 2000 isochrones/tracks.

3 . As the calibration algorithm is very flexible, new libraries can easily be produced as soon as better data and/or SED models are available. The present results, therefore, do not necessarily provide the final version of the BaSeL models.

4. As a first application, we are using the "Padova 2000 tracks" library for the analysis of the colours of chemo-evolutionary dynamical models of spiral galaxies (Westera 2001; Westera et al. 2001).

Acknowledgements. This work was supported by the Swiss National Science Foundation. 
Table 4. Magnitudes and colours for single stellar populations sythesised using the BaSeL 3.1 "Padova 2000" SED library.

\begin{tabular}{lrrrrrrrrrrrrr}
\hline age & {$[\mathrm{Fe} / \mathrm{H}]$} & $M_{\text {bol }}$ & $M_{V}$ & $U-B$ & $B-V$ & $V-I$ & $V-K$ & $R-I$ & $J-H$ & $H-K$ & $J-K$ & $K-L$ & $K-M$ \\
\hline 12.000 & 0.25 & 6.582 & 7.491 & 0.776 & 1.045 & 1.269 & 3.346 & 0.644 & 0.698 & 0.229 & 0.927 & 0.178 & 1.094 \\
14.000 & 0.00 & 6.616 & 7.407 & 0.599 & 0.978 & 1.224 & 3.130 & 0.634 & 0.691 & 0.200 & 0.892 & 0.164 & 1.020 \\
14.000 & -0.50 & 6.453 & 7.020 & 0.320 & 0.856 & 1.124 & 2.672 & 0.587 & 0.621 & 0.164 & 0.785 & 0.129 & 0.839 \\
18.000 & -1.00 & 6.523 & 6.976 & 0.155 & 0.771 & 1.051 & 2.366 & 0.532 & 0.555 & 0.135 & 0.691 & 0.120 & 0.735 \\
20.000 & -1.50 & 6.541 & 6.958 & 0.053 & 0.717 & 1.011 & 2.201 & 0.507 & 0.519 & 0.128 & 0.647 & 0.120 & 0.724 \\
20.000 & -2.00 & 6.563 & 6.978 & -0.009 & 0.684 & 0.980 & 2.135 & 0.498 & 0.499 & 0.121 & 0.620 & 0.116 & 0.731 \\
\hline
\end{tabular}

Table 5. Differences in colours between single stellar populations sythesised using the BaSeL 3.1 "Padova 2000" SED library and template globular cluster integrated energy distributions from Bica et al., in the sense BaSeL 3.1 - template.

\begin{tabular}{lrrrrr}
\hline template & {$[\mathrm{Fe} / \mathrm{H}]$} & $\Delta U-B$ & $\Delta B-V$ & $\Delta V-I$ & $\Delta R-I$ \\
\hline G177 & 0.25 & - & - & 0.034 & -0.009 \\
G1 - M31cl & 0.00 & - & -0.015 & -0.014 & -0.029 \\
G2b-M31cl & -0.50 & 0.024 & -0.013 & 0.007 & 0.002 \\
G3r & -1.00 & 0.062 & 0.032 & 0.007 & -0.017 \\
$G 4 b$ & -1.50 & 0.012 & 0.041 & -0.021 & -0.044 \\
$G 5$ & -2.00 & -0.008 & 0.045 & 0.032 & -0.009 \\
\hline
\end{tabular}

\section{References}

Alcaino, G. 1977, A\&AS, 29, 397

Alcaino, G., \& Liller, W. 1985, A\&A, 146, 389

Alcaino, G., \& Liller, W. 1986, AJ, 91, 87

Alcaino, G., Liller, W., Alvarado, F., et al. 1997, AJ, 114, 1067 Allard, F., \& Hauschildt, P. H. 1995, ApJ, 445, 433

Alonso, A., Arribas, S., \& Martínez-Roger, C. 1999, A\&AS, 140,261

Angelov, T. 1996, BOBeo, 153, 19

Arribas, S., \& Martínez-Roger, C. 1987, A\&A, 178, 106

Barmby, P., \& Huchra, J. P. 2000, ApJ, 531, L29

van den Bergh, S. 1988, AJ, 95, 106

Bessell, M. S. 1979, PASP, 91, 589

Bessell, M. S. 1983, PASP, 95, 480

Bessell, M. S., \& Brett, J. M. 1988, PASP, 100, 1134

Bessell, M. S., \& Weiss, E. W. 1987, PASP, 99, 642

Bica, E., Ortolani, S., \& Barbuy, B. 1994, A\&A, 106, 161

Bica, E., Alloin, D., Bonatto, C., et al. 1996 (private communication to T. Lejeune)

von Braun, K., Chiboucas, K., Minske, J. C., Salgado, J. F., \& Worthey, G. 1998, PASP, 110, 810

Brocato, E., Castellani, V., Poli, F. M., \& Raimondo, G. 2000, A\&AS, 146, 91

Bruzual, A. G. 1999, in Galaxy Interactions at Low and High Redshift, ed. J. E. Barnes, \& D. B. Sanders, IAU Symp., 186 (Dordrecht: Kluwer), 459

Bruzual, A. G., \& Charlot, S. 1993, ApJ, 405, 538

Bruzual, A. G., \& Charlot, S. 2000, Galaxy isochrone spectral synthesis evolution library (private communication)

Bruzual, A. G., Barbuy, B., Ortolani, S., et al. 1997, AJ, 114, 1531

Buser, R., \& Kurucz, R. L. 1992, A\&A, 264, 557

Cannon, R. D. 1974, MNRAS, 167, 551

Carretta, E., \& Gratton, R. G. 1997, A\&AS, 121, 95

Cathey, L. R. 1974, AJ, 791370

Charlot, S., \& Bruzual, A. G. 1991, ApJ, 367, 126
Cohen, J. G., Frogel, J. A., \& Persson, S. E. 1978, ApJ, 222, 165

daCosta, G. S., \& Armandroff, T. E. 1990, AJ, 100, 162

Demarque, P., \& McClure, R. D. 1977, ApJ, 213, 716

Drissen, L., \& Shara, M. M. 1998, AJ, 115, 725

Eggen, O. J. 1972, ApJ, 172, 639

Evans, T. L. 1983, SAAOC, 7, 86

Fagotto, F., Bressan, A., Bertelli, G., \& Chiosi, C. 1994, A\&AS, 105, 39

Ferraro, F. R., Carretta, E., Corsi, C. E., et al. 1997, A\&A, 320,757

Fricke, K. J., Izotov, Y. I., Papaderos, P., Guseva, N. G., \& Thuan, T. X. 2001, AJ, 121, 169

Frogel, J. A., Persson, S. E., Matthews, K., \& Aaronson, M. 1978, ApJ, 220, 75

Frogel, J. A., Persson, S. E., \& Cohen, J. G. 1981, ApJ, 246, 842

Frogel, J. A., Persson, S. E., \& Cohen, J. G. 1983, ApJ, 53, 713

Girardi, L., Bressan, A., Chiosi, C., Bertelli, G., \& Nasi, E. 1996, A\&AS, 117, 113

Girardi, L., Bressan, A., Bertelli, G., \& Chiosi, C. 2000, A\&AS, 141, 371 ("Padova 2000" isochrones)

González Delgado, R. M., García-Vargas, M. L., Goldader, J., et al. 1999, ApJ, 513, 707

Grebel, E. K., \& Roberts, W. J. 1995, A\&AS, 109, 293

Harris, W. E. 1996, AJ, 112, 1487

Hesser, J. E., \& Hartwick, F. D. A. 1977, ApJS, 33, 361

Hesser, J. E., Harris, W. E., VandenBerg, D. A., et al. 1987, PASP, 99, 739

Johnson, H. L., \& Sandage, A. R. 1956, ApJ, 124, 379

Johnson, J. A., \& Bolte, M. 1998, AJ, 115, 693

Johnson, K. E., Leitherer, C., Vacca, W. D., \& Conti, P. S. 2000, AJ, 120, 1273

Kaluzny, J. 1997, A\&AS, 122, 1

Kaluzny, J., Wysocka, A., Stanek, K. Z., \& Krzeminsky, W. 1998, Acta Astron., 48, 439

Kauffmann, G., \& Charlot, S. 1998, MNRAS, 294, 705

King, I. R., Anderson, J., Cool, A. M., \& Piotto, G. 1998, ApJ, 492, L37

Kong, X., Zhou, X., Chen, J., et al. 2000, AJ, 119, 2745

Kotilainen, J. K., Reunanen, J., Laine, S., \& Ryder, S. D. 2001, A\&A, 366, 439

Kurucz, R. L. 1995, private communication to R. Buser

Lastennet, E., Lejeune, T., Westera, P., \& Buser, R. 1999, A\&A, 341, 857

Lastennet, E., Valls-Gabaud, D., \& Oblak, E. 2000, in Birth and Evolution of Binary Stars, ed. B. Reipurth, \& H. Zinnecker, IAU Symp., 200 (Dordrecht: Kluwer), 164

Lastennet, E., Lignières, F., Buser, R., et al. 2001, A\&A, 365, 535

Lee, S. W. 1977, A\&AS, 27, 381 
Lee, S.-G., Lee, M. G., \& Kim, E. 1996, J. Korean Astron. Soc., 29, 171

Lee, H., Yoon, S., \& Lee, Y. 2000, AJ, 120, 998

Leitherer, C., Schaerer, D., Goldader, J. D., et al. 1999, ApJS, 123,3

Lejeune, T., Cuisinier, F., \& Buser, R. 1997, A\&AS, 125, 229 (Paper I)

Lejeune, T., Cuisinier, F., \& Buser, R. 1998, A\&AS, 130, 65 (Paper II)

Lejeune, T. 1997, Contribution à la synthèse spectrale évolutive d'amas et de galaxies par calibration de bibliothèques stellaires, Ph.D. Thesis, Univ. Strasbourg \& Univ. Basel, $334 \mathrm{pp}$.

Liu, M. C., Charlot, S., \& Graham, J. R. 2000, ApJ, 543, 644

Lotz, J. M., Ferguson, H. C., \& Bohlin, R. C. 2000, ApJ, 532, 830

Maraston, C., \& Thomas, D. 2000, ApJ, 541, 126

Marconi, G., Buonanno, R., Carretta, E., et al. 1998, MNRAS, 293,479

Marleau, F. R., Graham, J. R., Liu, M. C., \& Charlot, S. 2000, AJ, 120, 1779

Menzies, J. 1973, MNRAS, 163, 323

Mermilliod, J.-C. 1998, The General Catalogue of Photometric Data http://obswww . unige.ch/gcpd/

Mollá, M., \& García-Vargas, M. L. 2000, A\&A, 359, 18

Montegriffo, P., Ferraro, F. R., Fusi Pecci, F., \& Origlia, L. 1995, MNRAS, 276, 739

Newell, E. B., Rodgers, A. W., \& Searle, L. 1969, ApJ, 156, 597

Nikolaev, S., \& Weinberg, M. D. 2000, ApJ, 542, 804

Norris, J., \& Freeman, K. C. 1982, ApJ, 254, 143
Origlia, L., Goldader, J. D., Leitherer, C., Schaerer, D., \& Oliva, E. 1999, ApJ, 514, 96

Piotto, G., Cool, A. M., \& King, I. R. 1997, AJ, 113, 1345

Richer, H. B., \& Fahlman, G. G. 1987, ApJ, 316, 189

Ridgway, S. T., Joyce, R. R., White, N. M., \& Wing, R. F. 1980, ApJ, 235, 126

Rieke, G. H., \& Lebofsky, M. J. 1985, ApJ, 288, 618

Salaris, M. 2001, Theoretical uncertainties in Red Giant Branch stellar models, in Observed HR diagrams and stellar evolution: the interplay between observational constraints and theory, ed. T. Lejeune, \& J. Fernandes, ASP Conf. Ser., submitted

Salaris, M., Chieffi, A., \& Straniero O. 1993, ApJ, 414, 580

Sandage, A. 1969, ApJ, 157, 515

Sandage, A. 1970, ApJ, 162, 841

Sandage, A., \& Walker, M. F. 1966, ApJ, 143, 313

Sandquist, E. L., Bolte, M., Stetson, P. B., \& Hesser, J. E. 1996, ApJ, 470, 910

Saviane, I., Rosenberg, A., Piotto, G., \& Aparicio, A. 2000, A\&A, 355, 966

Scholz, M. 1997, private communication to R. Buser

Stetson, P. B., \& Harris, W. E. 1988, AJ, 96, 909

Westera, P. 2001, The BaSeL 3.1 models: Metallicity calibration of a theoretical stellar spectral library and its application to chemo-dynamical galaxy models, Ph.D. Thesis, Univ. of Basel, 378 pp.

Westera, P., Samland, M., Buser, R., \& Gerhard, O. E. 2001, A\&A, Colour Evolution of Disk Galaxy Models, submitted

Woolley, R., Alexander, J. B., Mather, L., \& Epps, E. 1961, Royal Obs. Bull., 43, 303 